\title{
Efecto de Supresión Eferente de las Emisiones Otoacústicas Transientes y Discriminación de Habla en Ruido
}

\author{
Efferent Suppression of Transient-evoked \\ Otoacoustic Emissions and Speech \\ Discrimination in Noise
}

Adrián Fuente $\mathbf{C}$. Unidad de Audiología, Escuela de Fonoaudiología, Facultad de Medicina, Universidad de Chile

Ximena Hormazábal R. Unidad de Audiología, Escuela de Fonoaudiología, Facultad de Medicina, Universidad de Chile

\footnotetext{
Alison López M. Unidad de Audiología, Escuela de Fonoaudiología, Facultad de Medicina, Universidad de Chile

Macarena Bowen M. Laboratorio de Audiología, Escuela de Fonoaudiología, Facultad de Medicina, Universidad de Chile
}

Contacto con el autor: Adrián Fuente C. (PhD) Escuela de Fonoaudiología, Universidad de Chile. Independencia 1027 Santiago-Chile. Tel: (56 2) 9786606 Fax: (56 2) 9786608 e-mail: afuente@med.uchile.cl

Recibido: Abril, 2010 Aceptado: Julio, 2010

\section{RESUMEN}

El tracto olivo-coclear medial ha sido ampliamente estudiado en animales de experimentación y clínicamente en humanos. Se ha sugerido que una de sus funciones se relaciona con la discriminación de habla en presencia de ruido de fondo. Actualmente no se cuenta con estudios sistemáticos de las posibles correlaciones entre el efecto de supresión eferente y el rendimiento en tareas de discriminación de habla en ruido. Por lo anterior, en nuestro Laboratorio estamos investigando los valores del efecto de supresión y cómo estos pueden relacionarse con tareas de discriminación de habla. En el presente artículo se reportan los resultados de un estudio piloto en esta materia. Un grupo de 45 sujetos adultos y auditivamente normales fueron estudiados. Los procedimientos incluyeron emisiones otoacústicas transientes, efecto de supresión de las emisiones otoacústicas transientes, reflejos acústicos ipsi y contralaterales (para las frecuencias $0.5,1,2$ y $4 \mathrm{kHz}$ ) y la prueba de HINT. No se encontraron diferencias estadísticamente significativas para el efecto de supresión eferente entre ambos oídos. Por otra parte, no se observaron correlaciones entre la amplitud del efecto de supresión y los valores obtenidos en la prueba de HINT para las subpruebas de habla en ruido. Se discuten los hallazgos encontrados y la necesidad de futuras investigaciones.

Palabras clave: Efecto de supresión eferente, emisiones otoacústicas, discriminación de habla en ruido, reflejos acústicos.

\begin{abstract}
The medial olivo-cochlear bundle has been widely studied in experimental animals and clinically, in human subjects. It has been suggested that one of the functions of this bundle relates to the discrimination of speech in the presence of background noise. No systematic studies of possible associations between the efferent suppression of otoacustic emissions (OAEs) and performance on speech-in-noise tasks has been carried out. For this reason, we are investigating the amplitude of the efferent suppression of OAEs and how this may be associated with performance on speech-in-noise tasks. The results of a pilot study on this topic are addressed in this manuscript. A total of 45 normal-hearing adult subjects were studied. Transient-evoked OAEs (TEOAEs), the efferent suppression of TEOAEs, ipsi and contralateral acoustic reflexes $(0.5,1,2$, and $4 \mathrm{kHz})$ were obtained and the hearing-in-noise-test (HINT) was administered to all the subjects selected. No significant differences were found in the efferent suppression of TEOAEs between ears. No significant correlations between results for the efferent suppression of TEOAEs and those for the HINT were found. These results and the need for further research are discussed.
\end{abstract}

Keywords: Efferent suppression, speech discrimination in noise, acoustic reflexes, otoacoustic emissions. 


\section{Introducción}

El tracto olivo-coclear medial genera un control eferente de las células ciliadas externas de la cóclea. Lo anterior se traduce en una regulación de las contracciones lentas generadas en estas células. Una forma clínica de evaluar este tracto es a través de la aplicación de un ruido contralateral durante la obtención de las emisiones otoacústicas ${ }^{1}$. Luego se compara la amplitud de las emisiones obtenidas durante la aplicación de ruido contralateral con la amplitud de las mismas obtenidas en ausencia de este ruido. Se espera observar que la amplitud de las emisiones otoacústicas se vea reducida cuando existe ruido contralateral.

Considerando que la edad lleva consigo una reducción en la actividad del sistema auditivo central debido a la degeneración de estructuras relacionadas a la audición, se han estudiado las variaciones del efecto de supresión de las emisiones otoacústicas transientes en relación a esta variable $e^{2,3}$. Así, se ha postulado que la amplitud de la supresión disminuye con la edad. Keppler et $a .^{3}$ estudiaron las variaciones en la amplitud del efecto de supresión de las emisiones otoacústicas en un grupo de sujetos de entre 20 a 79 años de edad, en quienes se controló estadísticamente las diferencias en los umbrales audiométricos. De acuerdo a los resultados, el efecto de supresión resultó estar mayormente correlacionado con la edad que con los umbrales audiométricos. Resultados similares han sido encontrados por De Oliveira et al. ${ }^{2}$

Debido a que se postula que una de las funciones del tracto olivo-coclear medial es mejorar la detección de señales en presencia de ruido de fondo ${ }^{4}$, este tracto ha sido ampliamente estudiado en poblaciones de sujetos con trastornos relacionados al procesamiento auditivo, lenguaje y lecto-escritura. Algunos de estos estudios han encontrado que la supresión de las emisiones otoacústicas es inferior en menores con trastornos de lenguaje y dificultades de discriminación en presencia de ruido de fondo en comparación a grupos controles de menores sin estas dificultades ${ }^{5}$. No obstante, otros estudios no han encontrado estas diferencias ${ }^{6}$. Por otra parte, en menores con alteraciones en el procesamiento auditivo (central) se ha encontrado una disminución en la amplitud del efecto de supresión y un aumento en los umbrales de los reflejos acústicos en comparación a menores $\sin$ alteraciones en el procesamiento auditivo ${ }^{7,8}$. Resultados similares han sido encontrados por Muchnik et al. ${ }^{9}$, quienes observaron amplitudes del efecto de supresión descendidas en un grupo de menores con trastorno en el procesamiento auditivo (central) en comparación a un grupo control pareado por edad y género.

Además, Garinis et al. $^{10}$, al estudiar la supresión eferente en sujetos adultos con y $\sin$ trastorno de aprendizaje, encontraron que en aquellos sujetos sin trastornos del aprendizaje existió una dominancia en la supresión del oído derecho (mayor supresión), mientras que en los sujetos con trastorno de aprendizaje existió dominancia del oído izquierdo.

A pesar de las investigaciones llevadas a 
cabo en relación a la función del sistema olivococlear eferente medial y la forma de estudiarlo clínicamente, esto es, a través del efecto de supresión eferente, actualmente la aplicabilidad clínica del estudio de este efecto es limitada. Lo anterior puede ser debido a que actualmente no se cuenta con estudios sistemáticos en relación al efecto de supresión eferente y su correlación con pruebas audiológicas tales como la discriminación de habla en ruido y umbrales de los reflejos estapedianos. Como ha sido sugerido, el sistema olivo-coclear medial tendría un rol importante en la detección y discriminación de habla en la presencia de ruido de fondo ${ }^{11,12}$. Por tanto se podría hipotetizar que un sujeto con mayor amplitud del efecto de supresión eferente tendería a detectar y/o discriminar de mejor forma los sonidos como el habla en la presencia de ruido que un sujeto con menor amplitud en el efecto de supresión eferente. No obstante, no existe evidencia científica al respecto. Además, no existen estudios que entreguen valores normativos en relación a la amplitud del efecto de supresión.

Debido a la necesidad clínica de contar con valores normativos del efecto de supresión, su posible correlación con los umbrales de los reflejos estapedianos, y con el rendimiento en tareas de discriminación de habla en ruido, el presente estudio tuvo por objetivo obtener evidencia científica en cuanto a estos dos últimos aspectos. Esto con el objetivo final de aportar evidencia científica en cuanto a la aplicabilidad clínica de la exploración del efecto de supresión eferente de las emisiones otoacústicas.

\section{Método}

\section{Sujetos}

Se evaluaron 45 individuos (19 hombres y 26 mujeres) entre 20 y 52 años de edad (promedio de edad 28.4, D.E. 6.9). Todos ellos eran hablantes nativos del español de Chile y presentaban niveles de audición normal (umbrales audiométricos iguales o mejores a $20 \mathrm{~dB} \mathrm{HL}$ para las frecuencias 250 a $8000 \mathrm{~Hz}$ ). Cada sujeto fue informado acerca de los procedimientos a ser utilizados y otorgó su consentimiento escrito para participar en el estudio.

\section{Procedimientos}

Los procedimientos de selección de la muestra y de evaluación para la obtención de los datos a ser analizados fueron llevados a cabo en una cabina audiométrica sonoamortiguada, en el Laboratorio de Audiología de la Escuela de Fonoaudiología de la Universidad de Chile. Para la otoscopía se utilizó un otoscopio Heine 2000. La audiometría tonal liminal se realizó con un audiómetro Interacoustics AC33 con fonos TDH-39P. Para la timpanometría y estudio de reflejos acústicos se utilizó un impedanciómetro Interacoustics AZ7. Las emisiones otoacústicas transientes se obtuvieron a través de un equipo portátil Echoport Plus (Otodynamics, Londres). El equipo estaba conectado a un computador de escritorio el cual tenía instalado el software ILO 88 OAE. Para la evaluación de la discriminación de habla en presencia de ruido de fondo se utilizó la prueba de Hearing-in-Noise Test -HINT ${ }^{13}$ con el módulo de oraciones correspondiente a español latinoamericano. 
A continuación se describen los procedimientos para seleccionar a los participantes.

1) Cuestionario. En él se incluyen preguntas acerca de historia de patología otológica, enfermedades neurológicas, antecedentes de exposición laboral a ruido y otros agentes ototóxicos. Se seleccionaron solo aquellos sujetos que no presentaran algunos o todos los antecedentes antes mencionados.

2) Otoscopía. Se aplicó para establecer que los sujetos no presentaran alteraciones patológicas visibles del canal auditivo externo y de la membrana timpánica.

3) Audiometría bilateral para tonos puros. Se efectuó en una cabina sonoamortiguada, con ruido ambiente no mayor a $30 \mathrm{~dB}(\mathrm{~A})$ y de acuerdo a las pautas de la ISO 8253-1 (1989) $)^{14}$. Se obtuvieron los umbrales audiométricos para los tonos puros de 250, 500, 1000, 2000, 3000, 4000, 6000 y 8000 $\mathrm{Hz}$ para conducción aérea. El procedimiento modificado de Hughson y Westake ${ }^{15}$ descrito por Carhart y Jerger ${ }^{16}$ fue utilizado para obtener los umbrales de audición. Sólo aquellos sujetos con umbrales iguales o inferiores a $20 \mathrm{~dB}$ HL en todas las frecuencias exploradas, fueron incluidos en la muestra. Las personas con diferencias entre ambos oídos en una o más frecuencias de $10 \mathrm{~dB}$ o más no fueron incluidas en la muestra ${ }^{17}$.

4) Impedanciometría. Se realizó timpanometría y estudio de reflejos acústicos. Para los reflejos acústicos, la estimulación comenzó a 50 dB HL y ulteriores incrementos en $5 \mathrm{~dB}$ fueron llevados a cabo hasta obtener una respuesta. El umbral del reflejo acústico se consideró como la intensidad a la cual se generó un cambio reproducible en la admitancia acústica ${ }^{18}$. Aquellos sujetos seleccionados para participar en el estudio, debieron presentar curvas tipo $\mathrm{A}^{19}$ bilateral en la timpanometría y tener reflejos acústicos contralaterales en las frecuencias 500, 1000 y $2000 \mathrm{~Hz}$.

Una vez seleccionados los participantes se procedió a completar el estudio de reflejos acústicos (reflejos ipsilaterales para las frecuencias 500, 1000, 2000 y $4000 \mathrm{~Hz}$, y el reflejo contralateral en 4000 $\mathrm{Hz}$ ). A continuación se procedió a la obtención de las emisiones otoacústicas transientes en ambos oídos con y sin efecto de supresión. Los estímulos fueron entregados a los oídos a través de una sonda para emisiones otoacústicas ILO para adultos tipo $\mathrm{B}^{20}$. Se utilizaron estímulos evocados de clicks rectangulares de 80 us los cuales fueron presentados a $80 \pm 2 \mathrm{~dB}$ peSPL. Estos estímulos fueron no lineares. La ventana de respuesta de tiempo fue fijada a $2.5-20 \mathrm{~ms}$ y el filtro pasa banda fue ajustado en el rango entre $500 \mathrm{~Hz}$ a $6 \mathrm{kHz}$. La presencia/ausencia de una respuesta de emisiones otoacústicas transientes fue determinada principalmente en base al análisis de la reproductibilidad de la forma de la onda completa resultante. Además, se analizaron las amplitudes en cada banda frecuencial. Para el estudio del efecto de supresión, un estímulo supresor de ruido blanco fue entregado al oído contralateral a una intensidad de $60 \mathrm{~dB} \mathrm{SPL}^{8}$. El ruido blanco fue generado por un audiómetro (Interacoustics AC33). Cada oído fue primero 
examinado sin la presencia de ruido contralateral, y a continuación, una vez que se obtuvo respuesta, manteniendo la sonda en el conducto auditivo externo, se entregó el ruido blanco al oído contralateral. A partir de este procedimiento se procedió a obtener una segunda respuesta de emisiones otoacústicas transientes. Finalmente, se procedió a estudiar la discriminación de habla en silencio y en presencia de ruido de fondo a través de la prueba HINT. Para calcular el umbral de reconocimiento de habla (SRT, a partir del inglés "speech recognition/reception threshold"), un set de oraciones en silencio fueron presentadas biauralmente a través de fonos TDH-39P (HINT SRT). Se les solicitó a los sujetos repetir cada oración escuchada. A continuación, con el objetivo de estudiar la discriminación de habla en presencia de ruido de fondo, se procedió a utilizar las subpruebas en ruido de la prueba de HINT. Para cada subprueba en ruido (tres en total), el sistema HINT calcula las relaciones señal/ruido (RSR) que el sujeto requiere para discriminar correctamente el $50 \%$ de las oraciones (SRT). La primera subprueba en ruido (HINT 1) consiste en la presentación a 0 grados azimuth de una lista de 20 oraciones y simultáneamente un ruido cuyo espectro frecuencial es el mismo al de las oraciones. Por tanto para esta condición o subprueba no existe separación espacial entre las oraciones y el ruido de fondo. Una vez que se han presentado las 20 oraciones y el sujeto las ha repetido, ya sea correcta o incorrectamente, se calcula automáticamente la RSR. La segunda subprueba en ruido (HINT 2), consiste en la presentación a 0 grados azimuth de una nueva lista de 20 oraciones y a 90 grados azimuth (oído derecho) de un ruido cuyo espectro frecuencial es el mismo al de las oraciones. Una vez que se han presentado las 20 oraciones y el sujeto las ha repetido, ya sea correcta o incorrectamente, se calcula automáticamente la RSR. La siguiente subprueba en ruido de HINT (HINT 3) consiste en la presentación a 0 grados azimuth de una nueva lista de 20 oraciones y a 270 grados azimuth (oído izquierdo) de un ruido cuyo espectro frecuencial es el mismo al de las oraciones. Una vez que se han presentado las 20 oraciones y el sujeto las ha repetido, ya sea correcta o incorrectamente, se calcula automáticamente la RSR. Finalmente se obtiene un valor ponderado de las tres subpruebas en ruido de HINT (HINT Pond).

\section{Resultados}

Inicialmente se realizó un análisis descriptivo de los valores obtenidos por el grupo de sujetos en estudio para los umbrales audiométricos, umbrales de reflejos acústicos, amplitud de las emisiones otoacústicas transientes, amplitud del efecto de supresión de las emisiones otoacústicas transientes y los valores de la prueba de HINT. Las Figuras 1 y 2 muestran la amplitud de las emisiones otoacústicas transientes del oído derecho e izquierdo, y la amplitud del efecto de supresión, respectivamente. Las Figuras 3 y 4 muestran los valores del SRT de la prueba HINT, y los valores de la RSR obtenidos en las subruebas de habla en ruido de la prueba de HINT, respectivamente. La Tabla 1 muestra los umbrales de los reflejos acústicos ipsi y contralaterales del oído derecho e izquierdo. 


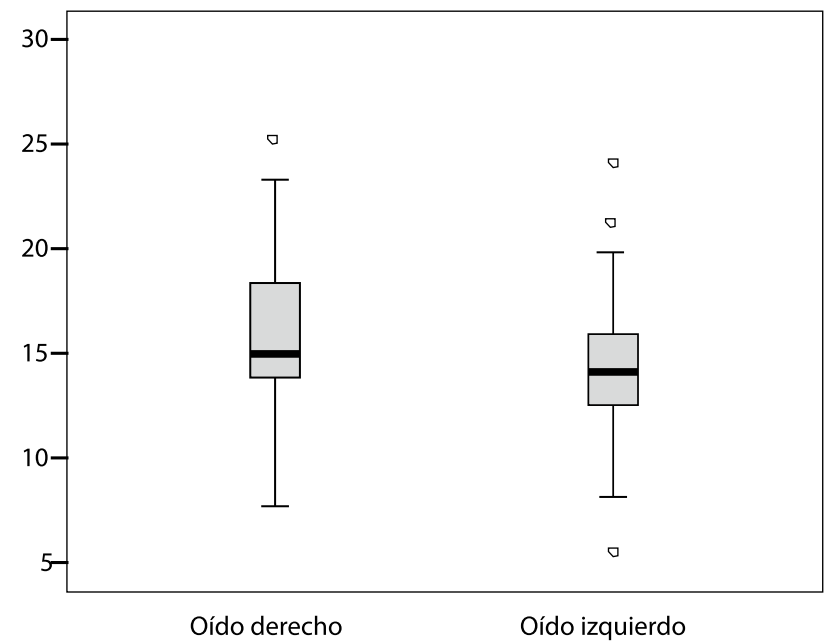

Figura 1. Boxplots de las amplitudes de las emisiones otoacústicas transientes del oído derecho e izquierdo obtenidas por los sujetos estudiados $(n=45)$. Los valores de las amplitudes están expresados en decibeles $(\mathrm{dB})$. Los rectángulos representan los valores del $50 \%$ de los casos. La línea trazada al interior de cada rectángulo representa los valores promedio. Los círculos representan los valores fuera de la norma y se definen como aquéllos que están ubicados entre 1.5 y 3 veces la longitud de cada rectángulo, medido desde el borde superior e inferior de éste.

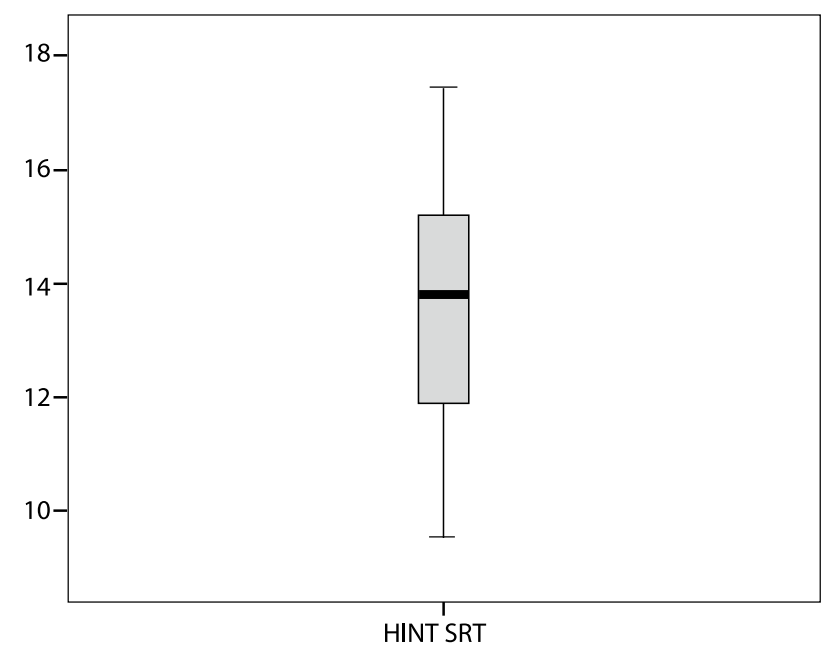

Figura 3. Boxplot de los valores del SRT obtenidos por los sujetos estudiados $(n=45)$ en la prueba de HINT. Los valores del SRT están expresados en decibeles (dB). El rectángulo representa los valores del $50 \%$ de los casos. La línea trazada al interior del rectángulo representa los valores promedio.

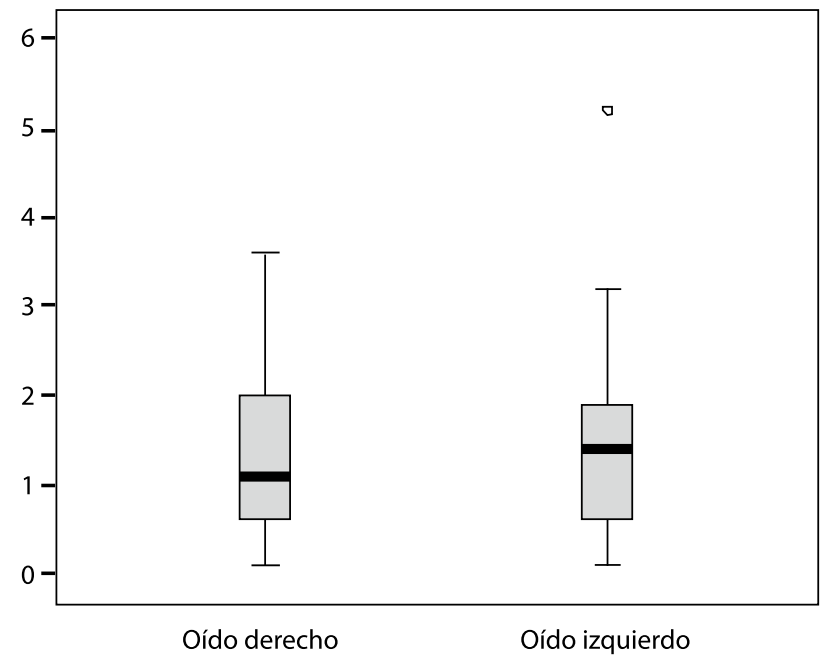

Figura 2. Boxplots de las amplitudes del efecto de supresión de las emisiones otoacústicas transientes del oído derecho e izquierdo obtenidas por los sujetos estudiados $(n=45)$. Los valores de las amplitudes están expresados en decibeles $(\mathrm{dB})$. Los rectángulos representan los valores del $50 \%$ de los casos. La línea trazada al interior de cada rectángulo representa los valores promedio. El círculo representa el valor fuera de la norma y se define como aquél que está ubicado entre 1.5 y 3 veces la longitud del rectángulo, medido desde el borde superior de éste.

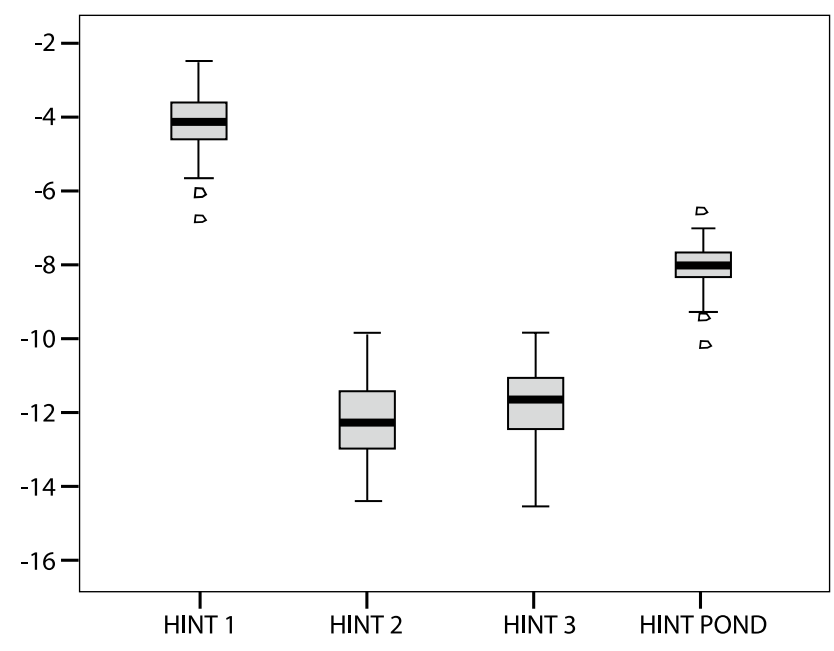

Figura 4. Boxplots de los valores de la RSR obtenidos por los sujetos estudiados $(n=45)$ en las subpruebas de habla en ruido de HINT. Los valores de la RSR están expresados en decibeles (dB). HINT 1: subprueba oraciones y ruido a 0 grado azimuth; HINT 2: subprueba oraciones a 0 grado azimuth y ruido a 90 grados azimuth; HINT 3: subprueba oraciones a 0 grado azimuth y ruido a 270 grados azimuth; HINT POND: valor ponderado de las tres subpruebas anteriores de HINT (HINT 1, 2 y 3). Los rectángulos representan los puntajes del 50\% de los casos. La línea trazada al interior de cada rectángulo representa los valores promedio. Los círculos representan los valores fuera de la norma y se definen como aquéllos que están ubicados entre 1.5 y 3 veces la longitud de cada rectángulo, medido desde el borde superior e inferior de éste. 
Tabla 1. Promedios y desviaciones estándares para los umbrales acústicos ipsi y contralaterales del oído derecho e izquierdo.

\begin{tabular}{ccc}
\hline Reflejo & Promedio & Desviación Estándar \\
\hline I Der $500 \mathrm{~Hz}$ & $85.0 \mathrm{~dB}$ & 19.3 \\
I Der $1000 \mathrm{~Hz}$ & $88.2 \mathrm{~dB}$ & 14.8 \\
I Der $2000 \mathrm{~Hz}$ & $89.2 \mathrm{~dB}$ & 15.0 \\
I Der $4000 \mathrm{~Hz}$ & $84.4 \mathrm{~dB}$ & 20.4 \\
C Der $500 \mathrm{~Hz}$ & $91.5 \mathrm{~dB}$ & 21.1 \\
C Der $1000 \mathrm{~Hz}$ & $92.1 \mathrm{~dB}$ & 21.2 \\
C Der $2000 \mathrm{~Hz}$ & $87.2 \mathrm{~dB}$ & 19.9 \\
C Der $4000 \mathrm{~Hz}$ & $87.8 \mathrm{~dB}$ & 21.3 \\
I Izq $500 \mathrm{~Hz}$ & $86.3 \mathrm{~dB}$ & 14.4 \\
I Izq $1000 \mathrm{~Hz}$ & $86.8 \mathrm{~dB}$ & 14.4 \\
I Izq $2000 \mathrm{~Hz}$ & $88.2 \mathrm{~dB}$ & 14.7 \\
I Izq $4000 \mathrm{~Hz}$ & $84.5 \mathrm{~dB}$ & 15.3 \\
C Izq $500 \mathrm{~Hz}$ & $94.0 \mathrm{~dB}$ & 16.1 \\
C Izq $1000 \mathrm{~Hz}$ & $93.0 \mathrm{~dB}$ & 15.6 \\
C Izq $2000 \mathrm{~Hz}$ & $88.7 \mathrm{~dB}$ & 14.9 \\
C Izq $4000 \mathrm{~Hz}$ & $86.6 \mathrm{~dB}$ & 20.6 \\
\hline
\end{tabular}

I: Ipsilateral C: Contralateral Der: Derecho Izq: Izquierdo

A través de la prueba de Wilcoxon se exploraron posibles diferencias entre el oído derecho e izquierdo, en el grupo de sujetos en estudio, para las amplitudes de las emisiones otoacústicas transientes y para el efecto de supresión de las emisiones otoacústicas transientes. Diferencias estadísticamente significativas se encontraron entre ambos oídos para las amplitudes de las emisiones otoacústicas $(Z=-5.84, p=0.00)$. A partir de la Figura 1 se observa que el promedio de la amplitud de las emisiones otoacústicas transientes es superior en el oído derecho (promedio $15.7 \mathrm{~dB}$ ) en comparación al oído izquierdo (promedio 14.4 dB). No se encontraron diferencias estadísticamente significativas entre los oídos para la amplitud del efecto de supresión de las emisiones otoacústicas transientes $(Z=-.88, p=.37)$. A partir de la Figura 2 , se observa una amplitud del efecto de supresión del oído derecho igual a $1.27 \mathrm{~dB}$, e igual a $1.44 \mathrm{~dB}$ en el oído izquierdo.
Por otra parte, debido a que el grupo de sujetos estuvo compuesto por individuos de ambos géneros, a través de la prueba de Mann-Whitney $\mathrm{U}$ se exploraron posibles diferencias en los valores obtenidos entre los géneros para la amplitud de las emisiones otoacústicas transientes, amplitud del efecto de supresión, y para todas las subpruebas de HINT (HINT SRT, HINT, 1, HINT 2, HINT 3, HINT ponderado). No se observaron diferencias estadísticamente significativas entre ambos géneros para ninguno de los resultados de las pruebas antes mencionadas.

Con el objetivo de establecer posibles correlaciones/asociaciones entre las variables en estudio: reflejos acústicos, discriminación de habla en silencioso y ruido, amplitud de las emisiones otoacústicas transientes, y amplitud del efecto de supresión de las emisiones otoacústicas transientes, a través de la prueba de Spearman se estudiaron 
posibles correlaciones entre estas variables. La variable edad también fue incluida en el análisis.

Las variables antes mencionadas fueron incorporadas en el análisis de la siguiente forma: reflejos acústicos (reflejos ipsilaterales para las frecuencias 500, 1000, 2000 y $4000 \mathrm{~Hz}$, para cada oído por separado y reflejos contralaterales para las frecuencias 500, 1000, 2000 y $4000 \mathrm{~Hz}$, para cada oído por separado); discriminación de habla en silencio (HINT SRT), discriminación de habla en ruido (HINT 1, HINT 2, HINT 3, HINT ponderado), amplitud de las emisiones otoacústicas transientes para cada oído por separado; y amplitud del efecto de supresión de las emisiones otoacústicas transientes para cada oído por separado.

Se encontró correlación estadísticamente significativa entre los resultados de la prueba de HINT SRT y la amplitud de las emisiones otoacústicas transientes del oído derecho $(R=-38$, $\mathrm{p}=.017)$, la amplitud de las emisiones otoacústicas del oído derecho e izquierdo $(\mathrm{R}=.40, \mathrm{p}=.006)$, y la amplitud de la supresión de las emisiones otoacústicas transientes del oído derecho e izquierdo $(R=.70, p=.00)$. Se encontró además correlación estadísticamente significativa entre el reflejo acústico contralateral del oído izquierdo a $2000 \mathrm{~Hz}$ y la amplitud del efecto de supresión de las emisiones otoacústicas transientes del oído izquierdo $(\mathrm{R}=-.341, \mathrm{p}=.022)$.

En relación a la edad, se encontró correlación estadísticamente significativa entre esta variable y el reflejo acústico ipsilateral del oído derecho a
$1000 \mathrm{~Hz}(\mathrm{R}=.33, \mathrm{p}=.025)$ e ipsilaterales del oído izquierdo a $500 \mathrm{~Hz}(\mathrm{R}=.405, \mathrm{p}=.006), 1000 \mathrm{~Hz}$ $(\mathrm{R}=.447, \mathrm{p}=.002)$, y $2000 \mathrm{~Hz}(\mathrm{R}=.300, \mathrm{p}=.045)$, así como también con los reflejos contralaterales del oído derecho a $500 \mathrm{~Hz}(\mathrm{R}=.357, \mathrm{p}=.016)$, y $1000 \mathrm{~Hz}(\mathrm{R}=.40, \mathrm{p}=.007)$, y contralateral del oído izquierdo a $500 \mathrm{~Hz}(\mathrm{R}=.404, \mathrm{p}=.006)$.

\section{Discusión}

A partir de la evaluación de 45 individuos adultos se estudió el efecto de supresión de las emisiones otoacústicas transientes y su posible relación con los umbrales de los reflejos estapediales y el desempeño en la discriminación de habla en ruido.

Según los resultados obtenidos, no se observó correlación entre el efecto de supresión y la discriminación de habla en ruido. Hemos mencionado enlaintroducción del presente artículo que una de las funciones sugeridas del sistema olivo-coclear eferente medial es precisamente el mejoramiento de la detección de señales en ruido y la discriminación de habla en ruido. Por tanto, se esperaría que una mayor amplitud en el efecto de supresión llevara consigo a un mejor rendimiento en tareas de discriminación de habla en ruido. Esto no fue observado en la presente investigación, no obstante, no descartamos el rol del sistema olivo-coclear eferente medial en las tareas de discriminación de habla en presencia de ruido de fondo.

El no haber encontrado correlaciones 
significativas entre ambas mediciones pudo haber sido debido a variables estadísticas, del diseño de la investigación, a la fluctuación de los valores de cada medición y a las características propias de la prueba de HINT. Se debe considerar que el tamaño de la muestra fue más bien pequeño y ello pudo haber influido en la ausencia de correlaciones significativas entre la amplitud del efecto de supresión y la prueba de HINT.

Por otra parte, la prueba de HINT corresponde a una prueba de discriminación de habla en ruido, la cual contiene material verbal altamente predictivo. Ello implica que el sujeto, al perder parte del mensaje, puede fácilmente cerrarlo a través del conocimiento del idioma. Por tanto, el rendimiento en esta prueba depende de la propia redundancia intrínseca del sistema auditivo así como de variables relacionadas más bien a los aspectos cognitivo-lingüísticos. Lo anterior se puede ejemplificar en que un sujeto puede no presentar un sistema eferente medial (SEM) totalmente normal/funcional, lo cual lo lleva a perder la mayor parte del mensaje verbal, versus un sujeto con un SEM totalmente normal, no obstante, debido a un mejor conocimiento lingüístico (influencias top down), el primer sujeto puede aun así discriminar mejor el mensaje verbal que el sujeto con un mejor funcionamiento a nivel del SEM.

En un estudio similar, Pollman, Hornsby $y$ Hood $^{21}$ no encontraron una asociación entre el SEM y tareas de percepción de habla en ruido. Los autores sugirieron que estos resultados podrían haber sido debido a la sensibilidad limitada de las pruebas utilizadas, a que las medidas del efecto de supresión presentan distintos mecanismos subyacentes, o bien debido a que influencias corticales pueden diferir entre las pruebas utilizadas. Sin embargo, algunos estudios han encontrado una asociación entre el sistema olivococlear eferente y tareas de discriminación de habla en presencia de ruido de fondo ${ }^{11,22}$.

Kumar y Vanja ${ }^{22}$ encontraron en un grupo de menores con buen desempeño escolar que el rendimiento en la percepción de habla con ruido ipsilateral a las relaciones señal ruido de +10 $\mathrm{dB} y+15 \mathrm{~dB}$ mejoraba cuando se presentaba un ruido contralateral. Además, este mejoramiento en la percepción de habla se correlacionó significativamente con la amplitud de la supresión contralateral de las emisiones otoacústicas.

De forma similar, Giraud et al. ${ }^{11}$ estudiaron la posible asociación entre el sistema olivococlear eferente y el rendimiento en tareas de discriminación de habla en ruido. Los autores compararon un grupo de sujetos postoperados con ablación de las vías auditivas eferentes y un grupo control. A ambos grupos se les presentó una tarea de discriminación de habla con ruido ipsilateral tanto en presencia y ausencia de ruido contralateral. Además se les evaluó la amplitud del efecto de supresión de las emisiones otoacústicas. Los resultados arrojaron que los sujetos controles mejoraban la discriminación de habla cuando existía un ruido contralateral. Esta mejoría, que fue casi inobservable en el grupo de sujetos con ablación de las vías eferentes, se correlacionó con los valores de la amplitud del efecto de supresión 
en los sujetos auditivamente normales. Los autores concluyeron que el sistema olivo-coclear eferente presenta un rol anti-enmascarador en la percepción de habla en ambientes ruidosos.

A diferencia de estos dos estudios, la presente investigación no estudió las diferencias para tareas de discriminación de habla en ruido en presencia y ausencia de un ruido contralateral. En el presente estudio solo abordamos tareas de discriminación de habla en presencia de ruido ipsilateral.

Los autores del presente artículo consideran que futuras investigaciones debieran utilizar una prueba de habla en ruido con un bajo nivel de predicción en el material verbal empleado para evaluar el funcionamiento del sistema olivococlear eferente medial. Pruebas que utilizan como material verbal monosílabos, o bien oraciones sintéticas, es decir, sin sentido semántico.

Además, de forma similar a otras investigaciones, el estudio de diferencias en el rendimiento para tareas de discriminación de habla en ruido en presencia y ausencia de un ruido contralateral debiera ser estudiado, además de posibles correlaciones entre la amplitud del efecto de supresión y la mejoría de la discriminación de habla en presencia de ruido contralateral.

\section{Referencias}

1. Di Girolamo, S.; Napolitano, B.; Alessandrini, M. \& Bruno, E. (2007) Experimental and clinical aspects of the efferent auditory system. Acta Neurochir Suppl, 97(Pt 2), 419-424.

2. De Oliveira, J.R.; Fernandes, J.C. \& Costa Filho, O.A. (2009) Age impact on the efferent system activates in cochlear mechanical properties in normal hearing individuals. Braz J Otorhinolaryngol, 75(3), 340-343.

3. Keppler, H.; Dhooge, I.; Corthals, P.; Maes, L.; D'haenens, W.; Bockstael, A.; Philips, B.; Swinnen, F. \& Vinck, B. (2010) The effects of aging on evoked otoacoustic emissions and efferent suppression of transient evoked otoacoustic emissions. Clin Neurophysiol, 121(3), 359-365.

4. Micheyl, C. \& Collet, L. (1996) Involvement of the olivocochlear bundle in the detection of tones in noise.J Acoust Soc Am, 99, 1604-1610.

5. Yalcinkaya, F.; Yilmaz, S.T. \& Muluk, N.B. (2010) Transient evoked otoacoustic emissions and contralateral suppressions in children with auditory listening problems. Auris Nasus Larynx, 37(1), 47-54.

6. Clarke, E.M.; Ahmmed, A.; Parker, D. \& Adams, C. (2006) Contralateral suppression of otoacoustic emissions in children with specific language impairment. Ear and Hearing, 27(2), 153-160.

7. Burguetti, F.A. \& Carvallo, R.M. (2008) Efferent auditory system: its effect on auditory processing. BrazJ Otorhinolaryngol, 74(5), 737-745.

8. Sanches, S.G. \& Carvallo, R.M. (2006) Contralateral suppression of transient evoked otoacoustic emissions in children with auditory processing disorder. Audiol Neurootol, 11(6), 366-372.

9. Muchnik, C.; Ari-Even Roth, D.; Othman-Jebara, R.; Putter-Katz, H.; Shabtai, E.L. \& Hildesheimer, M. (2004) Reduced medial olivocochlear bundle system function in children with auditory processing disorders. Audiol Neurootol, 9(2), 107-114.

10. Garinis, A.C.; Glattke, T. \& Cone-Wesson, B.K. (2008) TEOAE suppression in adults with learning disabilities. Int J Audiol, 47(10), 607-614.

11. Giraud, A.L.; Garnier, S.; Micheyl, C.; Lina, G.; Chays, A. \& Chéry-Croze, S. (1997) Auditory efferents involved in speech-in-noise intelligibility. Neuroreport, 8, 1779-1783. 
12. May, B.J.; Budelis, J. \& Niparko, J.K. (2004) Behavioral studies of the olivocochlear efferent system: learning to listen in noise. Arch Otolaryngol Head Neck Surg, 130, 660-664.

13. Nilsson, M.; Soli, S. D. \& Sullivan, J. A. (1994) Development of the Hearing in Noise Test for the measurement of speech reception thresholds in quiet and in noise. J Acoust Soc Am, 95, 1085-1099.

14. International organization for standardization (1989) Acoustic. Audiometrics test methods. Part 1: Basic pure tone air and bone conduction threshold audiometry. ISO 8253-1. ISO: Ginebra.

15. Hughson, W. \& Westlake, H. D. (1944) Manual for program outline for rehabilitation of aural casualties both military and civilian. Transactions of the American Academy of Ophthalmology and Otolaryngology, 48(Suppl.), $1-15$.

16. Carhart, R. \& Jerger, J. (1959) Preferred method for clinical determination of pure-tone thresholds. J Speech Hear Disord, 24, 330-345.
17. Keith, R.W. (1995) Development and standardization of SCAN-A: Test of auditory processing disorders in adolescents and adults. J Am Acad Audiol, 6, 286-292.

18. Freeman, S. \& Sohmer, H. (1990) The influence of sound stimulus parameters on the acoustic reflex waveform. Eur Arch Otorhinolaryngol, 247(2), 104-108.

19. Jerger, J. (1970) Clinical experience with impedance audiometry. Arch Otolaryngol, 92(4), 311-324.

20. Kemp, D.T.; Ryan, S. \& Bray, P. (1990) A guide to the effective use of otoacoustic emissions. Ear Hearing, 11(2), 93-105.

21. Pollman, M.; Hornsby, B. \& Hood, L.J. (2010) Efferent effects and speech understanding (abstract). Bulletin of the American Auditory Society, 35(1), 33.

22. Kumar, U.A. \& Vanja, C.S. (2004) Functioning of olivocochlear bundle and speech perception in noise. Ear Hearing, 25, 142-146. 